\title{
Flujos Isotérmicos/Térmicos, Incompresibles y Viscosos con Formulación Velocidad-Vorticidad
}

\author{
Blanca Bermúdez ${ }^{1}$ y Alfredo Nicolás. ${ }^{2}$ \\ (1) Benemérita Universidad Autónoma de Puebla, Facultad de Ciencias de la Computación, \\ Puebla-México (e-mail: bbj@solarium.cs.buap.mx) \\ (2) Universidad Autónoma Metropolitana, Departamento de Matemáticas, 09340 México D.F.-México \\ (e-mail: anc@xanum.uam.mx)
}

Recibido Jun. 22, 2009; Aceptado Ago. 13, 2009; Versión Final recibida Ago. 31, 2009

\begin{abstract}
Resumen
Se presentan resultados numéricos en dos dimensiones de flujos isotérmicos/térmicos de fluidos incompresibles y viscosos modelados matemáticamente por las ecuaciones de Navier-Stokes y por la aproximación de Boussinesq respectivamente en la formulación velocidad-vorticidad. Los flujos térmicos corresponden tanto a convección natural como a convección mixta. Los resultados se obtienen con un método simple el cual se basa en un proceso iterativo de punto fijo para resolver el sistema elíptico no lineal que resulta después de una discretización apropiada en el tiempo. El proceso iterativo lleva a la solución de problemas elípticos lineales, simétricos, bien condicionados y desacoplados para los cuales existen resolvedores eficientes sin importar la discretizacón espacial.
\end{abstract}

Palabras clave: fluidos viscosos, velocidad-vorticidad, flujo isotérmico, convección natural, discretización.

\section{Isothermal/Thermal Incompressible Viscous Fluid Flows with the Velocity-Vorticity Formulation}

\begin{abstract}
Numerical results in two dimensions for isothermal/thermal flows of viscous incompressible fluids mathematically modeled by the Navier-Stokes equations and the Bousssinesq approximation are presented in the velocity-vorticity formulation. The thermal flows correspond to natural and mixed convection. The results are obtained based on a fixed point iterative process for solving the nonlinear elliptic system that results after an appropriate time discretization is made. The iterative process leads to the solution of uncoupled, well conditioned linear elliptic problems for which very efficient solvers are known to exist regardless of the space discretization.
\end{abstract}

Keywords: viscous fluids, velocity-vorticity, isothermal flow, natural convection, dicretization. 


\section{INTRODUCCIÓN}

Se presentan resultados numéricos de flujos isotérmicos y térmicos de fluidos incompresibles y viscosos en 2 dimensiones, los cuales se modelan matemáticamente con las ecuaciones de NavierStokes y con la aproximación de Boussinesq no estacionarias respectivamente, considerando en este trabajo la formulación velocidad-vorticidad. Los flujos térmicos corresponden a convección natural y a convección mixta. Los resultados se obtienen con un método simple que se basa en un proceso iterativo de punto fijo para resolver el sistema elíptico no lineal que resulta una vez que se aplica una discretización adecuada en el tiempo. El proceso iterativo conduce a la solución de problemas elípticos, lineales, simétricos, bien condicionados y desacoplados para los cuales existen resolvedores eficientes, ya sea con diferencias finitas o con elementos finitos, mientras la región de flujo sea rectangular. Para el problema de la cavidad con tapa deslizable se presentan flujos isotérmicos y térmicos de convección mixta con números de Grashof $G r=100$ y números de Reynolds $\mathrm{Re}=400$ y 1000 ; para convección natural, donde todas las paredes de la cavidad son fijas, se reportan flujos para el número de Rayleigh $R a=10^{5}$. En todos estos casos se considera la razón geométrica $\mathrm{A}(\mathrm{A}=$ razón de la altura con el ancho) con $\mathrm{A}=1$ y $A \neq 1$.

Con variables velocidad y vorticidad es más difícil resolver estos flujos, al menos con un procedimiento numérico similar al aplicado en formulación función corriente-vorticidad para resolver el sistema elíptico no lineal análogo (Nicolás y Bermúdez, 2005; Baéz y Nicolás, 2006; Hoh-Minh et al., 2009), usando otra metodología (Davies y Carpenter, 2001), donde se presentan resultados con un esquema sofisticado para el caso de tres dimensiones. Aún más, trabajos sobre convección mixta son escasos (Iwatsu et al., 1993; Bermúdez y Nicolás, 1999), y más escasos todavía con velocidad y verticidad (Fusegi y Farouk, 1986; Arefmanesh et al., 2008).

Los modelos térmicos de convección natural y mixta tienen considerable aplicación en ingeniería. Respecto a los primeros se pueden mencionar, sistemas de almacenamiento de energía, aislamiento de reactores nucleares, ventilación de edificios (Le Quéré y Alziary de Roquefort, 1985; Baéz y Nicolás, 2006); respecto a los segundos, diseño de intercambiadores de calor (Iwatsu et al., 1993).

Todos los resultados corresponden a flujos en estado estacionario (límite para tiempos suficientemente grandes de la solución del problema no estacionario). Por otro lado, la contribución de este trabajo, después de un proceso de validación con $A=1$, consiste en presentar resultados con $A \neq 1$, con parámetros moderados, lo cual hasta ahora no es usual con variables velocidad-vorticidad.

\section{MODELO MATEMÁTICO Y MÉTODO NUMÉRICO}

Sea $\Omega \subset R^{N} \quad(N=2,3)$, la región de flujo de un fluido incompresible, viscoso, térmico y no estacionario y $\Gamma$ su frontera. Este tipo de fluidos está gobernado por el sistema de ecuaciones, no dimensional, en $\Omega \times(0, T), T>0$, dado por

$$
\left\{\begin{array}{l}
\mathbf{u}_{t}-\frac{1}{R e} \nabla^{2} \mathbf{u}+\nabla p+\mathbf{u} \cdot \nabla \mathbf{u}=\mathbf{f} \\
\nabla \cdot \mathbf{u}=0 \\
\theta_{t}-\frac{1}{\operatorname{RePr}} \nabla^{2} \theta+\mathbf{u} \cdot \nabla \theta=0
\end{array}\right.
$$

conocido como la aproximación de Boussinesq en variables primitivas, velocidad $\boldsymbol{u}$ y presión $\mathrm{p}$, si $\boldsymbol{f}=\frac{G r}{R e^{2}} \theta \boldsymbol{e}$ donde $\theta$ es la temperatura del flujo, y $\boldsymbol{e}$ es el vector unitario en la dirección gravitacional g. Los parámetros adimensionales $R e, G r$ y $P r$ son los números de Reynolds, Grashof y Prandtl respectivamente; $G r$ también puede expresarse como $\frac{R a}{P r}$, donde $R a$ es el número de Rayleigh. 
Estos parámetros están dados por $R e=\frac{U L}{v}$, con $v=\frac{U L}{R e}$ la viscosidad cinemática, $\mathrm{Ra}=\frac{\beta r^{3} x g \rho_{0}^{2}}{\mu^{3} c_{p}}\left(T_{1}-T_{0}\right), \operatorname{Pr}=k / \mu c_{p} g$ es la constante gravitacional y las temperaturas de referencia $T_{0}$ y $T_{1}, T_{0}<T_{1}$, las cuales pueden ser las temperaturas sobre las paredes verticales si la región de flujo es rectangular, $L$ y $U$ son la longitud y velocidad de referencia, incluyendo también coeficientes térmicos: $\mu$ viscosidad dinámica y $c_{p}$ el calor específico. La temperatura adimensional $\theta$ y la velocidad $u$ están dadas por $\theta=\frac{T-T_{0}}{T_{1}-T_{0}}$ y $\quad \mathbf{u}=\mathbf{u} / U$, y la posición adimensional $\boldsymbol{x}$ por $\mathbf{x}=\mathbf{x} / L$. Si el flujo no depende de la temperatura el acoplamiento con (1c) se elimina y $\mathrm{f}$ no depende de $\theta$, entonces (1a) y (1b) dan las Ecuaciones de Navier-Stokes para flujos isotérmicos. Es conveniente señalar que para convección mixta los parámetros que determinan el flujo son Re y Gr mientras que para convección natural es Ra. El sistema debe complementarse con condiciones iniciales y de frontera apropiadas Digamos $\mathbf{u}(\boldsymbol{x}, 0)=\mathbf{u}_{0}$ y $\theta(\boldsymbol{x}, 0)=\theta_{0}$ en $\Omega ; \boldsymbol{u}=\boldsymbol{f}$ y $B \theta=0$ sobre $\Gamma, t \geq 0$, con $B$ un operador de frontera para la temperatura que puede involucrar condiciones de tipo Dirichlet, Neumann o mixtas.

Tomando el rotacional en ambos lados de (1a) la ecuación para la vorticidad $\omega$ en $\Omega \times(0, T)$ resulta ser

$\omega_{\mathrm{t}}-\frac{1}{\operatorname{Re}} \nabla^{2} \omega+\mathbf{u} \cdot \nabla \omega=\omega \cdot \nabla \mathbf{u}+\mathrm{f}$,

donde el vector vorticidad está dado por

$\boldsymbol{\omega}=\nabla \times \mathbf{u}$.

Tomando el rotacional en (3) usando la identidad $\nabla \times \nabla \times \boldsymbol{a}=-\nabla^{2} \boldsymbol{a}+\nabla(\nabla \cdot \boldsymbol{a})$ y (1b), resulta una ecuación de Poisson para la velocidad

$\nabla^{2} \mathbf{u}=-\nabla \times \boldsymbol{\omega}$

Luego entonces, las ecuaciones (2), con $\mathrm{f}$ dependiente de $\theta$ como se dijo anteriormente, y (4), ambas acopladas a (1c), dan las aproximación de Boussinesq en variables velocidad y vorticidad.

Puede verificarse fácilmente que la ecuación de transporte escalar para la vorticidad $\omega$ en $\Omega \times(0, \mathbf{T}), \Omega \subset \mathbf{R}^{2}$, está dada por

$\omega_{\mathrm{t}}-\frac{1}{\operatorname{Re}} \nabla^{2} \omega+\mathbf{u} \cdot \nabla \omega=\frac{G r}{\operatorname{Re}^{2}} \frac{\partial \theta}{\partial x}$

Aún más, por la restricción bidimensional en (3),

$\omega=\frac{\partial \mathbf{u}_{2}}{\partial \mathbf{x}}-\frac{\partial \mathbf{u}_{1}}{\partial \mathbf{y}}$

$y$, de (4), se obtienen dos ecuaciones de Poisson para las componentes de la velocidad

$\left\{\begin{array}{l}\nabla^{2} u_{1}=-\frac{\partial \omega}{\partial y} \\ \nabla^{2} u_{2}=\frac{\partial \omega}{\partial x}\end{array}\right.$ 
Entonces, el sistema de aproximación vectorial de Boussinesq (2) y (4) acoplado a (1c) se reduce a un sistema escalar de cuatro ecuaciones en 2 dimensiones, uno dado por (5) y dos por (7), acoplados a (1c); (5) y (7) están relacionadas a través de (6). De (6), la condición de frontera para $\omega$ en (5) debe obtenerse de la dada para $\boldsymbol{u}=\left(u_{1}, u_{2}\right)$.

Para aproximar las derivadas temporales que aparecen en la ecuación para la vorticidad (5) y la ecuación para la temperatura (1c) se usa la siguiente aproximación de segundo orden

$f_{t}(x,(n+1) \Delta t) \approx \frac{3 f^{n+1}-4 f^{n}+f^{n-1}}{2 \Delta t}$

donde $x \in \Omega, n \geq 1, \Delta t$ denota el paso en el tiempo, y $f^{r} \approx f(x, r \Delta t)$, suponiendo que $f$ es suficientemente suave.

Entonces, aplicando esta aproximación en (5), (7), y (1c), en forma totalmente implícita, se obtiene el siguiente sistema elíptico no lineal en cada nivel de tiempo $(n+1) \Delta t$, en $\Omega$

$\begin{cases}\nabla^{2} u_{1}=-\frac{\partial \omega}{\partial \mathbf{y}} & \\ \nabla^{2} u_{2}=\frac{\partial \omega}{\partial \mathbf{x}}, & \mathbf{u}=\mathbf{u}_{\mathbf{b c}} \text { sobre } \boldsymbol{\Gamma} \\ \alpha \omega-\mathbf{v} \nabla^{2} \omega+\mathbf{u} \cdot \nabla \omega=\frac{\mathbf{G r}}{\mathbf{R e}^{2}} \frac{\partial \theta}{\partial \mathbf{x}}+\mathbf{f} \omega, & \omega=\omega_{\mathbf{b c}} \text { sobre } \boldsymbol{\Gamma} \\ \alpha \theta-\gamma \nabla^{2} \theta+\mathbf{u} \cdot \nabla \theta=\mathbf{f} \theta, & \mathbf{B} \theta=0 \text { sobre } \boldsymbol{\Gamma}\end{cases}$

donde $\alpha=\frac{3}{2 \Delta t}, f_{\omega}=\frac{4 \omega^{2}-\omega^{n-1}}{2 \Delta t}, f_{\theta}=\frac{4 \theta^{2}-\theta^{n-1}}{2 \Delta t}, \gamma=\frac{1}{\operatorname{Pr} \operatorname{Re}} y \frac{1}{\operatorname{Re}}$ se ha reemplazado por el

coeficiente de viscosidad cinemática $v$, con $U=L=1 ; \mathbf{u}_{b c}$ y $\omega_{b c}$ denotan la condición de frontera para u y $\omega$, y $B$ la de $\theta$, como se dijo antes. Este esquema de 3 niveles de tiempo requiere el valor de las variables en el nivel de tiempo 1, lo cual se obtiene resolviendo el sistema también de la forma (8), mediante una sucesión de niveles de tiempo usando un paso de tiempo menor, que resulta de aproximar las derivadas con un esquema de Euler de primer orden.

Puesto que el sistema (8) es tipo no potencial, puede usarse un proceso iterativo de punto fijo, el cual pude ser visto como una adaptación de uno para convección mixta en la formulación función corriente-vorticidad (Nicolás y Bermúdez, 2005), ó bien como una extensión a convección mixta de aquél para flujos isotérmicos en variables velocidad y verticidad (Nicolás y Bermúdez, 2007).

Si se denota

$R_{\omega}(\omega, \mathbf{u}) \equiv \alpha \omega-v \nabla^{2} \omega+\mathbf{u} \cdot \nabla \omega-\frac{G r}{\operatorname{Re}^{2}} \frac{\partial \theta}{\partial x}-f_{\omega} \quad$ y $\quad R_{\theta}(\theta, \mathbf{u}) \equiv \alpha \theta-\gamma \nabla^{2} \theta+\mathbf{u} \cdot \nabla \theta-f_{\theta}$, el sistema (8) es equivalente, en $\Omega$, a

$\begin{cases}\nabla^{2} u_{1}=-\frac{\partial \omega}{\partial \mathbf{y}} & \\ \nabla^{2} u_{2}=\frac{\partial \omega}{\partial \mathbf{x}}, & \mathbf{u}=\mathbf{u}_{\mathbf{b c}} \text { sobre } \boldsymbol{\Gamma} \\ R_{\theta}(\theta, \mathbf{u})=0, & \mathbf{B} \theta=0 \text { sobre } \boldsymbol{\Gamma} \\ R_{\omega}(\omega, \mathbf{u})=0, & \omega=\omega_{\mathbf{b c}} \text { sobre } \boldsymbol{\Gamma}\end{cases}$ 
Entonces, (9) se resuelve, en cada nivel de tiempo $(n+1)$, con el proceso iterativo de punto fijo siguiente. Con $\omega^{0}=\omega^{n}$ y $\theta^{0}=\theta^{n}$ dados, resolver hasta convergencia con respecto a $\theta$ y $\omega$ en $\Omega$

$$
\begin{aligned}
& \left\{\begin{array}{l}
\nabla^{2} u_{1}^{m+1}=-\frac{\partial \omega^{m}}{\partial y} \\
\nabla^{2} u_{2}^{m+1}=\frac{\partial \omega^{m}}{\partial x},
\end{array}\right. \\
& \theta^{m+1}=\theta^{m}-\rho_{\theta}(\alpha I-\gamma \Delta)^{-1} R \theta\left(\theta^{m}, \mathbf{u}^{(m+1)}\right), \quad B \theta^{m+1}=0 \text {, sobre } \Gamma \\
& \omega^{m+1}=\omega^{m}-\rho_{\omega}\left(\alpha I-v \nabla^{2}\right)^{-1} R \theta\left(\omega^{m}, \mathbf{u}^{(m+1)}, \theta^{m+1}\right), \omega^{m+1}=\omega_{b c}^{m} \text {, sobre } \Gamma, \rho_{\omega}>0
\end{aligned}
$$

Luego, tomar $\left(\mathrm{u}_{1}^{\mathrm{n}+1}, \mathrm{u}_{2}^{\mathrm{n}+1}, \theta^{\mathrm{n}+1}, \omega^{\mathrm{n}+1}\right)=\left(\mathrm{u}_{1}^{\mathrm{m}+1}, \mathrm{u}_{2}^{\mathrm{m}+1}, \theta^{\mathrm{m}+1}, \omega^{\mathrm{m}+1}\right)$.

Finalmente, el sistema (10) es equivalente, en $\Omega$, a

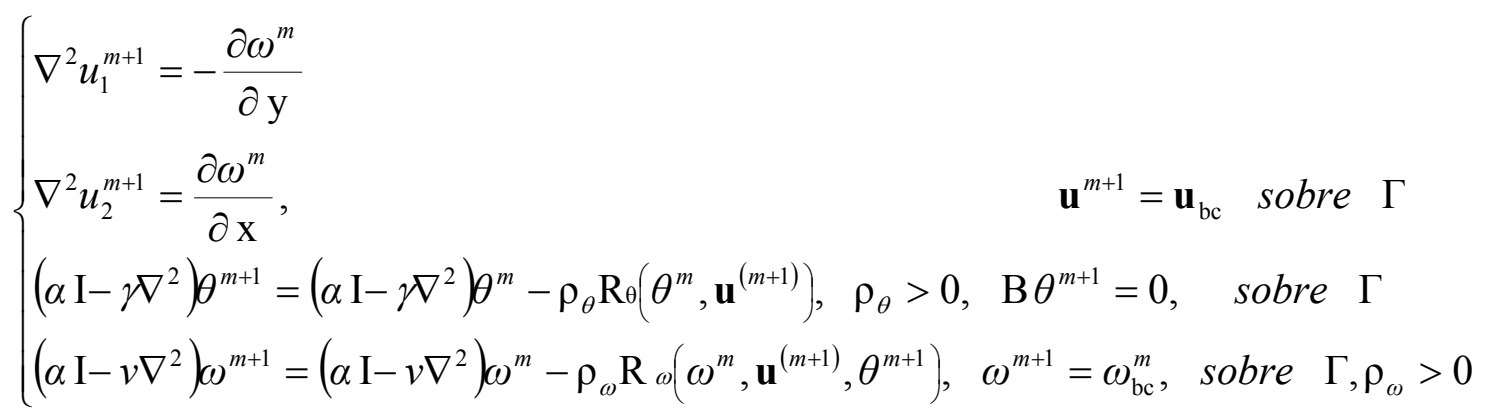

Luego entonces, en cada iteración, de cada nivel de tiempo $(n+1) \Delta t$, se tienen que resolver cuatro problemas elípticos, lineales, simétricos y desacoplados, asociados a los operadores $\alpha I-\gamma \nabla^{2} \mathrm{y}$ $\alpha I-v \nabla^{2}$ y dos con $\nabla^{2}$. Es claro que para flujos isotérmicos, el problema de la temperatura asociado con el operador $\alpha I-\gamma \nabla^{2}$ se elimina desde (1c) y a partir de ahí se considera $\mathrm{f}=0$. Debe notarse que la parte no simétrica para $\theta$ y $\omega$ en (8) se ha pasado al lado derecho, gracias al proceso iterativo. Para la discretización espacial de problema elípticos lineales, se pueden usar diferencias finitas ó elementos finitos mientras se trate de dominios de flujo rectangulares; en uno u otro caso, existen resolvedores eficientes. En el caso de elemento finito, se deben escoger formulaciones variacionales y luego restringirlas a espacios de elementos finitos de dimensión finita, por ejemplo aquellas formulaciones que se usan en Gunzburger (1989) y Glowinski (2003). Para los resultados específicos que se presentan aquí se hace uso de la aproximación de segundo o de cuarto orden del resolvedor Fishpack (Adams et al., 1980).

\section{EXPERIMENTOS NUMÉRICOS Y RESULTADOS}

Desde el trabajo puramente isotérmico en Nicolás y Bermúdez (2007) se ha señalado que contrariamente al caso de la formulación función corriente-vorticidad en Nicolás y Bermúdez (2005), donde también se usa un proceso de punto fijo después de la discretización temporal y la discretización espacial solamente usa aproximaciones de $2^{\circ}$ orden, en el caso de velocidadvorticidad, en general, hay que usar discretización espacial de $4^{\circ}$ orden; de hecho, para todos lo resultados que se reportan aquí se hace uso de la opción de $4^{\circ}$ orden en Adams et al. (1980), para resolver problemas elípticos.

Los experimentos numéricos, para flujos isotérmicos y de convección mixta, se llevan a cabo en cavidades rectangulares $\Omega=(0, a) \times(0, b), a, b>0$, con el problema de la cavidad con tapa deslizable, lo cual implica que la condición de frontera de u está dada por $\mathbf{u}=(1,0)$ en la frontera que se desliza $y=b$ y por $\mathbf{u}=(0,0)$ en cualquier otro lado; para convección natural $\mathbf{u}=(0,0)$ en todas las paredes, por 
viscosidad y por ser paredes sólidas y fijas. Se supone que la cavidad esta llena con aire, entonces el número de Prandtl es $P r=0.72$; luego, el flujo dependerá de $R e, G r, R a$ y de $A$ solamente. Los números de Reynolds, de Grashof, de Rayleigh y $A$ que se consideran en este trabajo son: $400 \leq R e \leq 1000, G r \leq 100, R a=10^{5} \quad y \frac{1}{2} \leq A \leq 2$. El flujo parte del reposo, entonces, la condición inicial para $\boldsymbol{u}$ y $\theta$ está dada por $\mathbf{u}(\boldsymbol{x}, 0)=0$ y $\theta(\boldsymbol{x}, 0)=0$ Los tamaños de malla se denotan por $h_{x}, h_{y}$ y el tamaño de paso de tiempo por $\Delta t$; los cuales se especifican en cada caso de estudio. Los resultados que se presentan son flujos en estado estacionario y se reportan a través de las líneas de corriente y los isocontornos de la vorticidad para flujos isotérmicos y a través de líneas de corriente e isotermas para flujos de convección mixta y natural. Los flujos de convección mixta se complementan con su transferencia de calor a través del número de Nusselt local $N u(x)$ y global $\overline{N u}$.

Por otro lado, para la condición de frontera para la temperatura (expresada dentro del operador B en (8)) en cada caso térmico bajo estudio se tiene

convección natural $\left\{\begin{array}{l}\theta=1 \text { sobre }\left.\Gamma\right|_{x=0}, \quad \theta=0 \text { sobre }\left.\Gamma\right|_{x=a} \\ \frac{\partial \theta}{\partial n}=0 \text { sobre }\left.\Gamma\right|_{y=0, b}\end{array}\right.$

lo cual significa que las paredes horizontales son adiabáticas y las verticales están a temperatura constante, y el calentamiento ocurre en la pared izquierda

convección mixta $\left\{\begin{array}{lll}\frac{\partial \theta}{\partial n}=0 & \text { sobre }\left.\Gamma\right|_{x=0, a} \\ \theta=\theta_{0} & \text { sobre }\left.\Gamma\right|_{y=0}, \quad \theta=\theta_{1} \equiv 1 \text { sobre }\left.\Gamma\right|_{y=b}\end{array}\right.$

lo cual significa que la pared de arriba (pared caliente), $(x, y)=(x, b)$, se mantiene a temperatura mayor que la de abajo (pared fría), $(x, y)=(x, 0)$, y las paredes laterales, $(x, y)=(0, y) y(x, y)=(a, y)$, están aisladas. Luego, el fluido se mueve por flotación del gradiente de temperatura vertical y por la condición de frontera en la tapa deslizable.

El número de Nusselt local $N u$ mide la transferencia de calor en cada punto de la pared caliente, donde la temperatura está especificada, y el número de Nusselt global $\overline{N u}$ el promedio de dicha transferencia; y están definidos por

Número de Nusselt local: $N u(x)=\left.\frac{\partial \theta}{\partial y}\right|_{y=b}$,

Número de Nusselt Global $\left.\overline{N u}\right|_{y=b}=\frac{1}{A} \int_{0}^{a} N u(x) d x$ :

La descripción de los resultados se presenta a continuación. Hasta donde se sabe, casi todos los resultados con $A \neq 1$ se están reportando por primera vez. Los resultados con $A=1$ se presentan con fines de validación.

Flujos isotérmicos. Las figuras 1 y 2 muestran flujos isotérmicos para $R e=400$ con $A=1$ y 2 respectivamente; las mallas respectivas son $\left(h_{x}, h_{y}\right)=(1 / 100,1 / 100)$ y $\left(h_{x}, h_{y}\right)=(1 / 120,2 / 240)$ y los tamaños de paso en el tiempo $\Delta t=0.01 \quad y \quad \Delta t=0.001$. El resultado con $A=2$ coincide bastante bien con el reportado en Bruneau y Jouron (1990) y Goyon (1996), ambos trabajos con variables distintas 
de velocidad y vorticidad y usando otra metodología, y cualitativamente, con su respectiva escala, con el de $R e=1000$ en Nicolás y Bermúdez (2005). Con $A=1$, respecto a las líneas de corriente, el vórtice central y el número de subvórtices, 2, coinciden perfectamente con Ghia et al. (1982) y bastante bien respecto a los contornos de vorticidad; en Sellountos y Sequeira (2008) solamente aparece un subvórtice pero la vorticidad es un poco mejor, comparan también con Ghia et al. (1982).
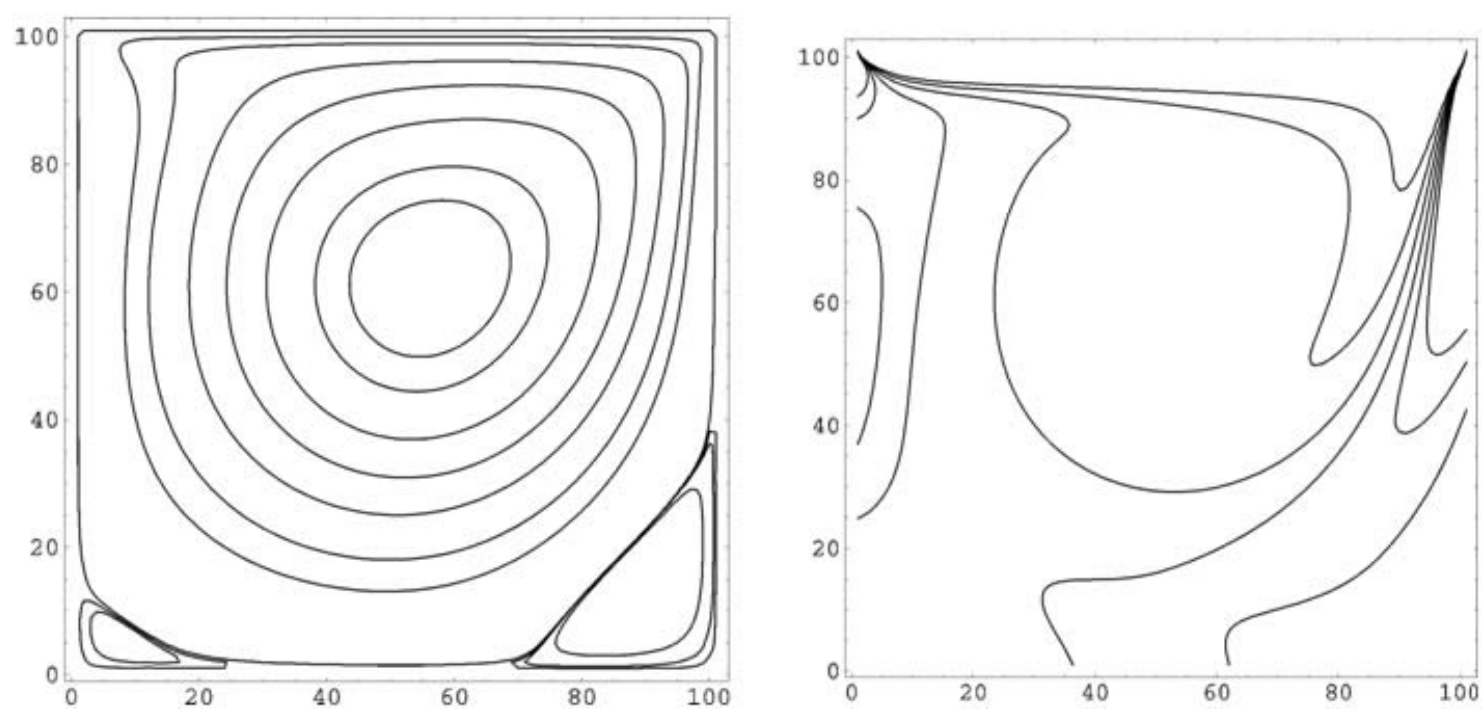

Fig.1: $R e=400,\left(h_{x}, h_{y}\right)=\left(\frac{1}{100}, \frac{1}{100}\right)$, líneas de corriente (izq.), iso-vorticidad (der.)

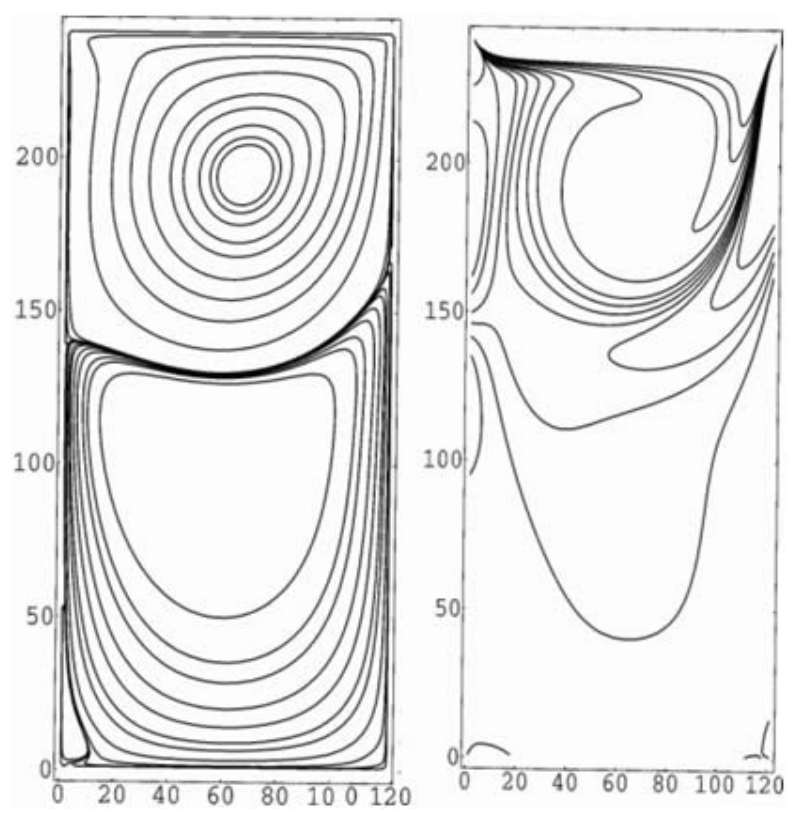

Fig. 2: $\operatorname{Re}=400,\left(h_{x}, h_{y}\right)=\left(\frac{1}{120}, \frac{2}{240}\right)$, líneas de corriente (izq.), iso-vorticidad (der.)

Flujos térmicos de convección natural. Las figuras 3 y 4 muestran flujos de convección natural para $R a=10^{5} \quad$ con $A=1$ y 2 respectivamente; las mallas respectivas $\operatorname{son}\left(h_{x}, h_{y}\right)=(1 / 64,1 / 64) y(1 / 64,2 / 128)$ y los tamaños de paso en el tiempo, $\Delta t=0.0001 \mathrm{y}$ 0.00001 . A este respecto, la Tabla 1 muestra resultados para $R a=10^{5}$ para comparar con De Vahl Davis (1983), cuyos resultados son obtenidos mediante un método de transitorio falso. La comparación se lleva a cabo con $\left.\psi\right|^{\text {mid }}$ valor absoluto de $\psi$ en el centro de la cavidad y con el número de Nusselt global $\overline{N u}$. Puede observarse que para $A=1$ dichos valores están cerca con los 
de De Vahl Davis (1983) a pesar del hecho de que los métodos son diferentes; en dicho trabajo los resultados para $A=2$ no son reportados, lo cual se indica en la Tabla 1 mediante *'s. Hacemos notar que con $A=1$, las isotermas y las líneas de corriente, excepto dos contornos casi circulares dentro de la línea de más adentro que se obtienen con ciertos valores especificados, coinciden perfectamente con los de De Vahl Davis (1983); lo mismo se puede decir respecto al resultado en Hoh-Minh et al (2009), en este trabajo $\overline{N u}=4.521$. En dicha Tabla también se observa que la transferencia de calor global disminuye si $A$ se incrementa, lo cual coincide con lo que ocurre con la formulación función corriente-vorticidad.

Flujos térmicos de convección mixta. La figura 5, muestra el flujo para $R e=400$ y $G r=100$ con $A=1$, el cual se obtiene con $\left(h_{x}, h_{y}\right)=(1 / 128,1 / 128)$ y $\Delta t=0.001$. Este resultado coincide cualitativamente, dentro de la respectiva escala, con el reportado Nicolás y Bermúdez (2005) y Bermúdez y Nicolás (1999) para $R e=1000$ y $G r=100$. Las figuras 6 y 7 describen los flujos para los mismos $R e$ y $G r$ con $A=2$ y $A=1 / 2$; en estos casos, $\Delta t=0.001$ y las respectivas mallas están dadas por $\left(h_{x}, h_{y}\right)=(1 / 128,2 / 256)$ y $\left(h_{x}, h_{y}\right)=(2 / 256,1 / 128)$. Para estos casos no se conocen otros resultados con los cuales comparar, sin embargo, dada la baja influencia de $G r$ es razonable que, cualitativamente, las líneas de corriente sean similares a las de los casos isotérmicos y que la deformación de las isotermas sea congruente con lo que se observa con $A=1$. En relación a transferencia de calor, la figura 8 muestra dicha transferencia a través de $N u(x)$ en la pared de arriba: $(x, y)=(x, 1), 0 \leq x \leq 2$ para $A=1 / 2 ;(x, y)=(x, 1), 0 \leq x \leq 1$ para $\mathrm{A}=1 ; \mathrm{y}(x, y)=(x, 2), 0 \leq x \leq 1$ para $A=2$. Las gráficas muestran que la transferencia de calor crece a medida que $A$ decrece; lo cual es congruente con los valores del número de Nusselt global $\overline{N u}$ dados por 11.645, 3.698 y 0.636 para $A=1 / 2,1$ y 2 respectivamente. Por otro lado, en la figura 8 también se observa que el valor máximo de $N u(x)$ ocurre muy cerca de $x=0$ mientras que el mínimo ocurre en $x=1$ para $\mathrm{A}=1$ y 2 , y en $x=2$ para $A=1 / 2$

Tabla 1. $R a=10^{5}: \psi^{\text {mid }}$ vs. $\psi_{\text {Vahl Davis }}^{\text {mid }}, \overline{N u}$ vs. $\overline{N u}_{\text {Vahl Davis }}$

\begin{tabular}{|c|c|c|c|c|}
\hline$A$ & $|\psi|^{\text {mid }}$ & $|\psi|_{\text {Vahl Davis }}^{\text {mid }}$ & $\overline{N u}$ & $\overline{N u}_{\text {Vahl Davis }}$ \\
\hline 1 & 15.111 & 11.97 & 4.577 & 4.531 \\
\hline 2 & 31.414 & ${ }^{* * *}$ & 4.350 & $* * *$ \\
\hline
\end{tabular}
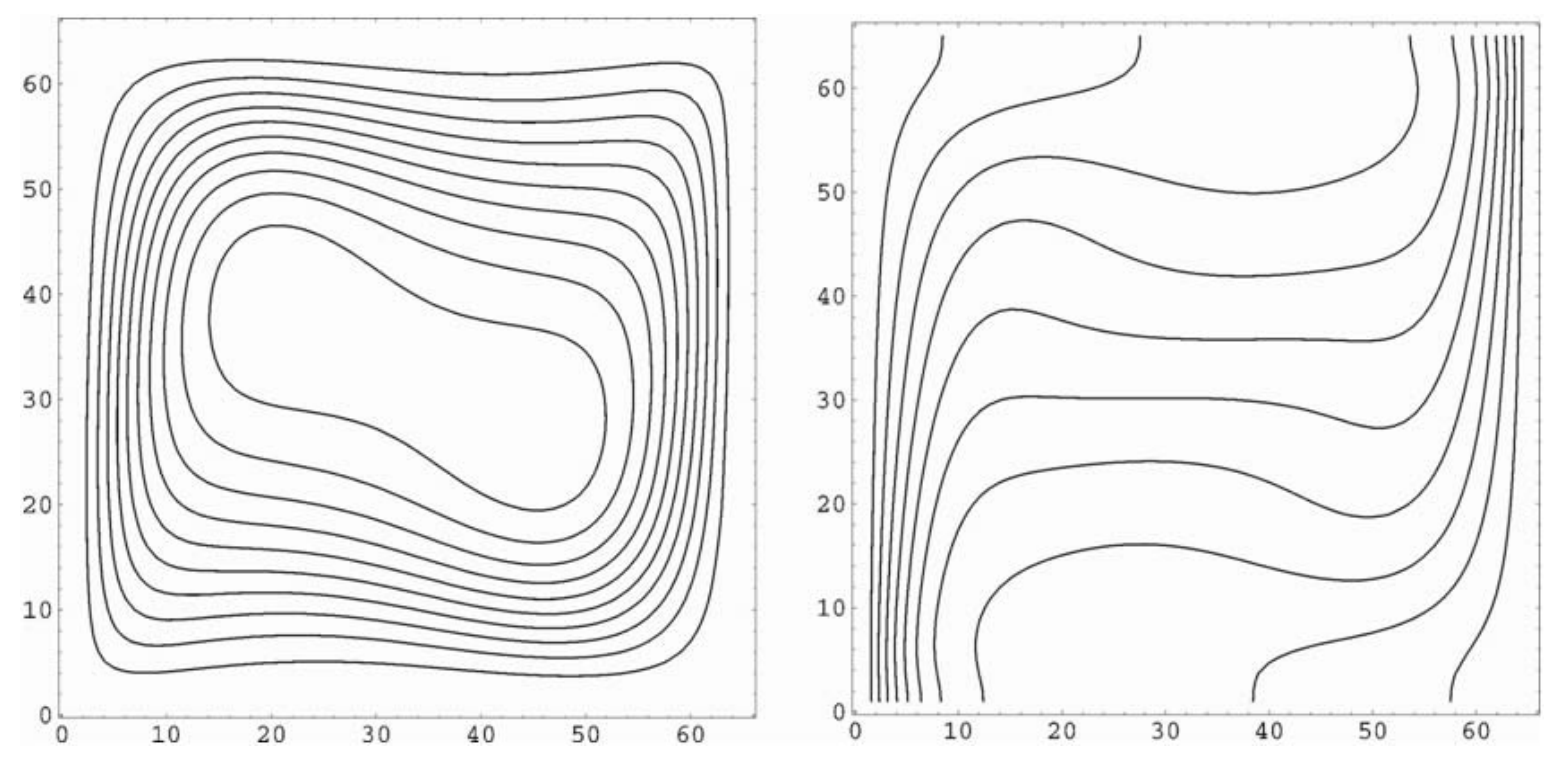

Fig. 3: $R a=100000,\left(h_{x}, h_{y}\right)=\left(\frac{1}{64}, \frac{1}{64}\right)$, líneas de corriente (izq.), isotermas (der.) 

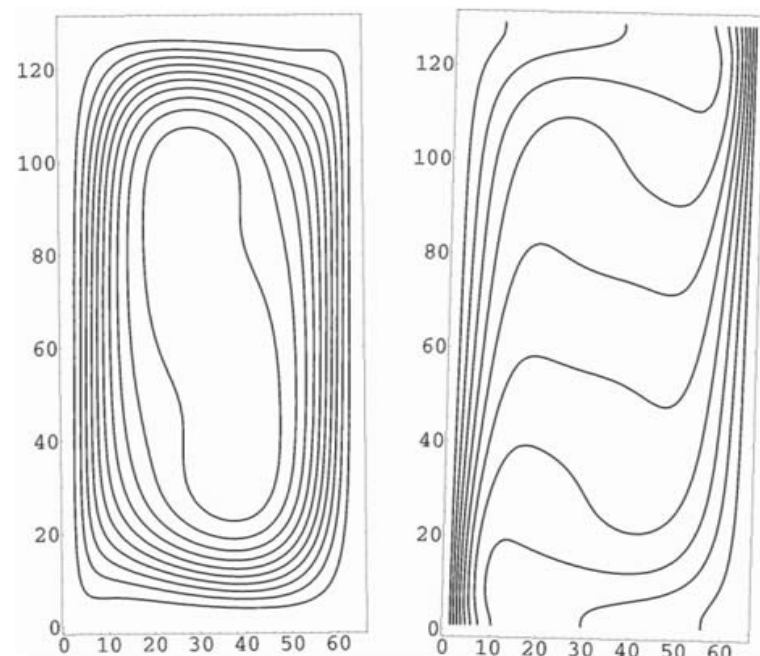

Fig. 4: $R a=100000,\left(h_{x}, h_{y}\right)=\left(\frac{1}{64}, \frac{2}{128}\right)$, líneas de corriente (izq.), isotermas (der.)
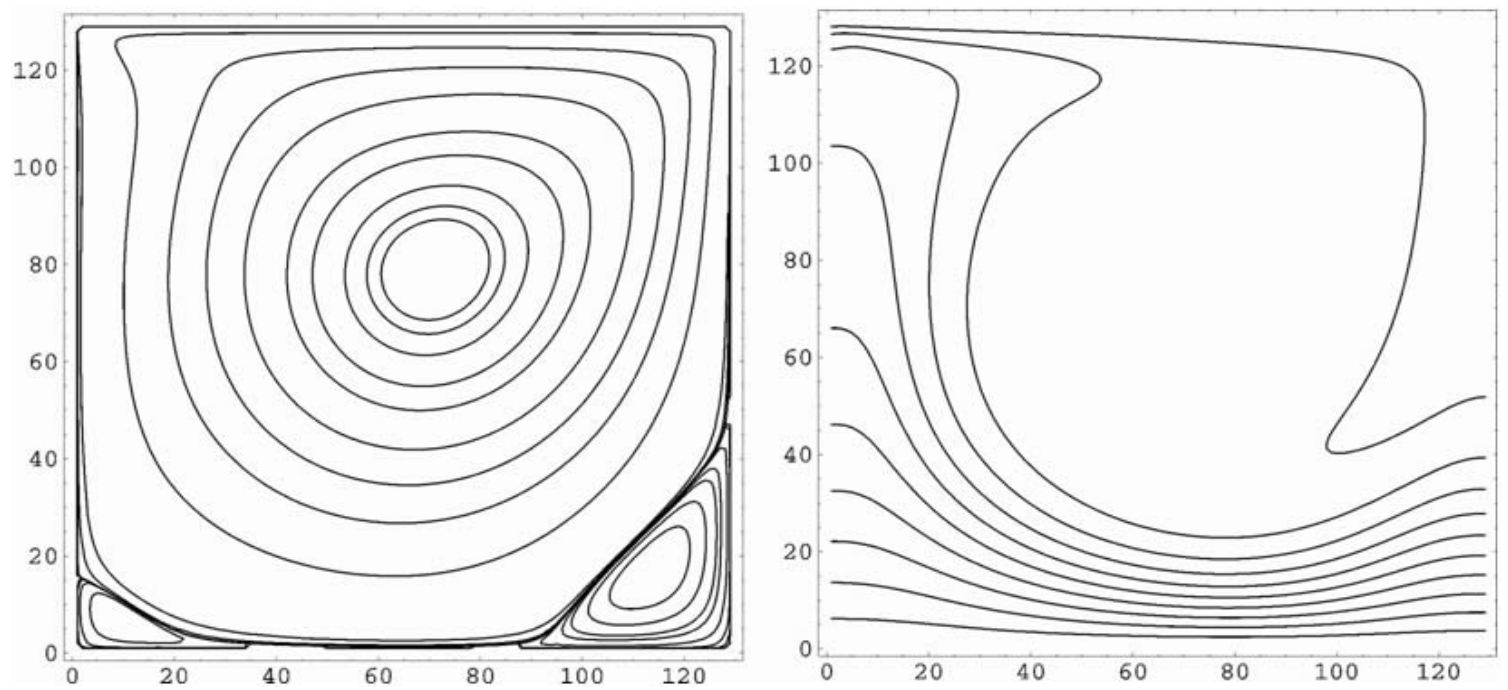

Fig. 5: $R e=400, G r=100,\left(h_{x}, h_{y}\right)=\left(\frac{1}{128}, \frac{1}{128}\right), A=1$; líneas corriente (izq.), isotermas (der.)
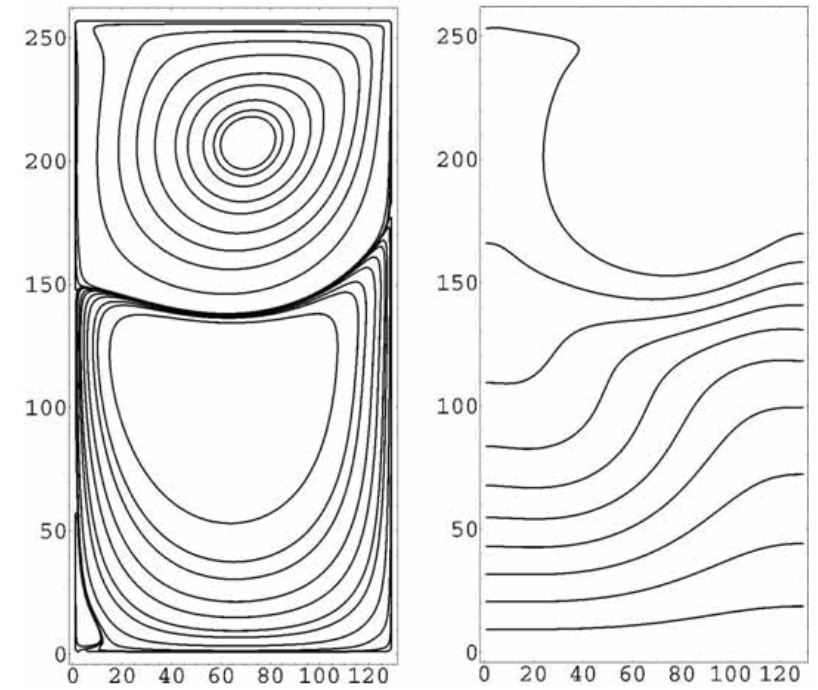

Fig. $6 R e=400, G r=100,\left(h_{x}, h_{y}\right)=\left(\frac{1}{128}, \frac{2}{256}\right), A=2$; líneas corriente (izq.), isotermas (der.)
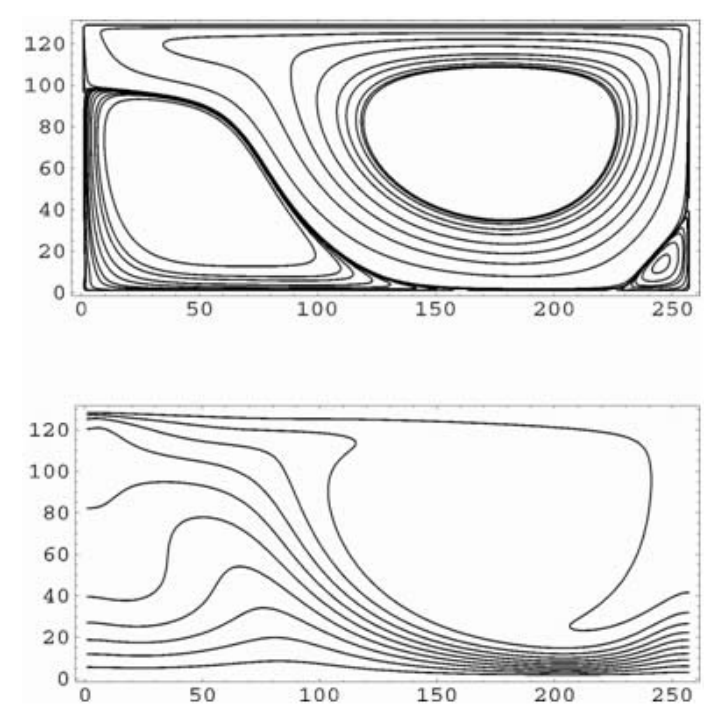

Fig. 7: $\mathbf{R e}=400, G r=100,\left(\mathbf{h}_{\mathbf{x}}, \mathbf{h}_{y}\right)=\left(\frac{2}{256}, \frac{1}{128}\right), \mathbf{A}=\frac{1}{2}$; líneas de corriente (arriba), isotermas (abajo) 


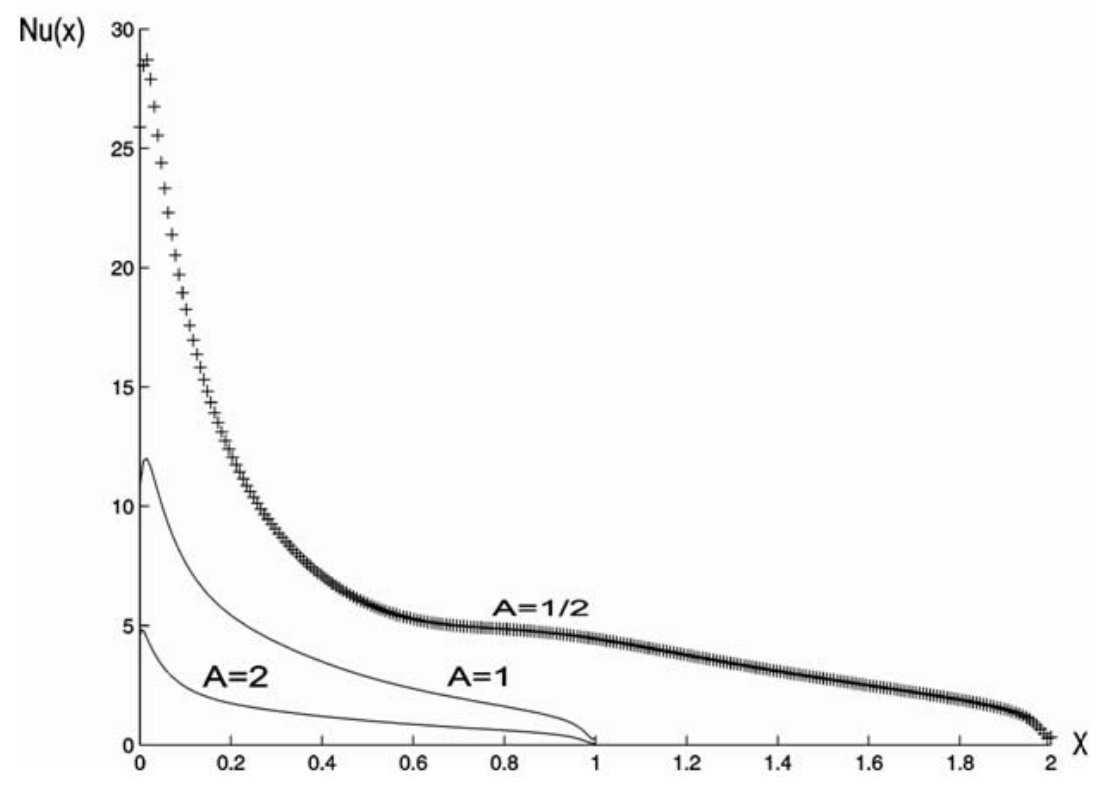

Fig. 8: Nusselt locales para $\mathbf{A}=1,2 \quad y \frac{1}{2}$

\section{CONCLUSIONES}

Se han presentado resultados numéricos para flujos de fluidos incompresibles y viscosos tanto isotérmicos como térmicos de convección natural y mixta los cuales se modelan matemáticamente por las ecuaciones de Navier-Stokes y la aproximación de Bousinessq en la formulación velocidadvorticidad. Obtener dichos flujos con esta formulación y con un método numérico muy cercano a uno que se ha aplicado a la formulación función corriente- vorticidad no es del todo fácil. Por lo cual, los resultados que aquí se reportan consideran solamente números de Reynolds y de Grashof bastante moderados para los casos isotérmico y de convección mixta, y números de Rayleigh también moderados para convección natural; sin embargo, con ellos se logra suficiente efectividad para ser capaces de variar el aspecto geométrico de la cavidad A, lo cual se sabe que ocasiona que el flujo , desde el caso isotérmico, se vuelva más inestable, Nicolás y Bermúdez (2005), Bruneau y Jouron (1990), Goyon (1996).

\section{REFERENCIAS}

Adams, J., P. Swarztrauber y R. Sweet; FISHPACK: A package of fortran subprograms for the solution of separable elliptic PDE's, The National Center of Atmospheric Research, Boulder, Colorado USA (1980).

Arefmanesh, A., M. Najafi y H. Abdi; Meshless local Petrov Galerkin Method with Unity Test Function for Non-Isothermal Fluid Flow. Computer Modeling in Engineering and Sciences: 25(1), 9-22 (2008)

Báez, E., A. Nicolás; 2D natural convection flows in tilted cavities: porous media and homogeneous fluids. Int. J. of Heat and Mass Transfer: 49, 4773-4785 (2006)

Bermúdez, B. y A. Nicolás; An efficient scheme for Thermal/Isothermal Incompressible Viscous Flow, Int. J. Num. Meth. Fluids: 29, 397-410 (1999).

Bruneau, C.H. y C. Jouron; An efficient scheme for solving steady incompressible Navier-Stokes equations, Journal of Computational Physics: 89, 289-413 (1990).

Davies Christopher y P.W. Carpenter; A Novel Velocity-Vorticity Formulation of the Navier-Stokes Equations with Applications to Boundary Layer Disturbance Evolution, Journal of Computational Physics: 172, 119-165 (2001). 
De Vahl Davis, G.; Natural Convection of air in a square cavity: a benchmark numerical solution, Int J. Numer. Methods Fluids:, 3, 2496-264 (1983).

Fusegi, T. y B. Farouk; Predictions of Fluid Flow and Heat Transfer Problems by Vorticity-Velocity Formulation of the Navier Stokes Equations, J. of Computational Physics: 65, 227-243 (1986).

Ghia, U., K.N. Ghia y C.T. Shin; High-Re solutions for incompressible flow using the Navier-Stokes equations and a multigrid method. J. of Computational Physics: 48, 387-411 (1982).

Glowinski R.; Handbook of Numerical Analysis: Numerical Methods for Fluids (Part. 3). North-Holland Ed. (2003).

Goyon, O.; High-Reynolds number solutions of Navier- Stokes equations using incremental unknowns. Computer Methods in Applied Mechanics and Engineering: 130, 319-335 (1996).

Gunzburger, M.D.; Finite Element Methods for Viscous Incompressible Flows: A guide to theory, practice, and algorithms, Academic Press, INC (1989).

Ho-Minh D., N. Mai-Duy y T. Tran-Cong; Galerkin-RBF Approach for the Streamfunction-VorticityTemperature Formulation of Natural Convection in 2D Enclosured Domains, Computer Modeling in Engineering and Sciences: 44(3), 219-248 (2009).

Iwatsu, R., Min Hyn Jae, y Kuwahara Kunio; Mixed convection in a driven cavity with a stable vertical temperature gradient, Int. J. Heat and Mass Transf.: 36, 1601-1608 (1993)

Le Quéré, P. y T. Alziary de Roquefor; Computation of Natural Convection in Two-Dimensional Cavities with Chebyshev Polynomials. J. of Computational Physics: 57, 210-228 (1985).

Nicolás, A. y B. Bermúdez; 2D thermal/isothermal incompressible viscous flows, Int. J. Numer. Meth. Fluids: 48, 340-366 (2005).

Nicolás, A. y B. Bermúdez; 2D Viscous incompressible flows by the velocity-vorticity Navier-Stokes equations, Computer Modelling in Engineering and Sciences: 20(2), 73-83 (2007).

Sellountos, A.J. y A. Siqueira; A Hybrid Multi-Region BEM/LBIE-RBF Velocity-Vorticity Scheme for the Two-Dimensional Navier-Stokes Equations, Computer Modeling in Engineering and Sciences: 23(2), 127-147 (2008). 